\title{
DIRECT NUMERICAL SIMULATIONS OF SMALL PARTICLES IN TURBULENT FLOWS OF LOW DISSIPATION RATES USING ASYMPTOTIC EXPANSION
}

\author{
Sandipan Banerjee, ${ }^{1}$ Orlando Ayala, ${ }^{2}$ Lian-Ping Wang ${ }^{3,4, *}$ \\ ${ }^{1}$ University of Louisville, Department of Mechanical Engineering, Louisville, KY 40292, USA \\ ${ }^{2}$ Old Dominion University, Department of Engineering Technology, Norfolk, VA 23529, USA \\ ${ }^{3}$ Southern University of Science and Technology, Department of Mechanics and Aerospace \\ Engineering, Shenzhen, Guandong 518055, China \\ ${ }^{4}$ University of Delaware, Department of Mechanical Engineering, Newark, DE 19711, USA
}

\begin{abstract}
Collisions of sedimenting droplets in a turbulent flow is of great importance in cloud physics. Collision efficiency and collision enhancement over gravitational collision by air turbulence govern the growth of the cloud droplets leading to warm rain initiation and precipitation dynamics. Due to the low flow dissipation rate in stratocumulus clouds, a related challenge is low droplet Stokes number. Here the Stokes number is the ratio of droplet inertial response time to the flow Kolmogorov time. A very low Stokes number implies that the numerical integration time step is now governed by the droplet inertial response time, rather than the time step necessary for the flow simulation. This situation makes a simulation very expensive to perform. With the motivation to speed up the simulations, we implement an asymptotic expansion approach for particle tracking as this method is suitable for low particle Stokes number and avoids the numerical integration of the stiff equation of motion of droplets. We first validate our implementation using the simpler 2-D cellular flow. Next, we compare the collision statistics of the newly implemented asymptotic approach with our existing approach of particle tracking as well as with published results from the literature. Finally, we provide the run time comparison for both methods. We report that for $10 \mu \mathrm{m}$ and $20 \mu \mathrm{m}$ particle radius, in a flow of dissipation rate $\epsilon=10 \mathrm{~cm}^{2} / \mathrm{s}^{3}$, asymptotic expansion approach achieves a speed-up by a factor of 25.2.
\end{abstract}

KEY WORDS: Turbulence, DNS, collision, cloud droplets

\section{INTRODUCTION}

\subsection{Motivation}

Particle laden turbulent flows are commonly found in both natural as well as industrial flows; raindrop growth in the clouds, aerosol processing, and pneumatic transport of solids are some examples. In these flows, the rate of coagulation is determined by the geometric collision rate, the collision efficiency, and the coagulation efficiency. The geometric collision is affected by the carrier turbulent flow due to the effects of turbulence on the relative motion and the local distributions of the inertial particles ([1],[2],[3]). The collision efficiency is affected by the local particle-particle hydrodynamic interactions due to disturbance flows induced by the particles ([2],[3]). The coagulation efficiency is governed by interfacial forces such as the surface tension and van der Waals force, etc. This paper focuses on raindrop growth in clouds by collision-coalescence,

*Corresponding Lian-Ping Wang 3,: lwang@udel.edu 
specifically for stratocumulus clouds where the viscous dissipation rate is relatively low. Our main concern is the geometric collision rates and collision efficiency of cloud droplets.

Since the air motion in clouds is of turbulent nature, there have been many studies on the effect of turbulence in the enhancement of collision-coalescence which relates the intensity of air turbulence and particle inertia to the increase of collision-coalescence. Some of these studies were in qualitative agreement ([4-6]) and some were in disagreement on the theory of turbulence enhancing the collision rate [7]. On the quantitative level, there had been no general consensus until relatively recently. The recent advance is largely due to rigorous simulation studies by $[1,3,8]$, who provide evidence of turbulence enhancing the collision rate. For engineering applications, the turbulence dissipation rate is higher compared to that of cloud droplet background flow. A lower dissipation rate corresponds to a larger Kolmogorov time and thus smaller particle Stokes numbers, now that the particle inertial response time determines the time step size while the statistics must still be computed based on the flow time scales, therefore direct numerical integration of the particle equation of motion becomes computationally expensive. For a combination of weak turbulence and small particle response time, here we test an alternative approach using an asymptotic expansion approach [9]. The implementation has been validated by comparing the particle trajectories in a 2-d cellular flow and then in a turbulent flow. Finally, results for collision statistics using the asymptotic expansion method are reported.

\subsection{Background}

In this work we study collision of cloud droplets by direct numerical simulations where the background turbulent flow is generated by solving the incompressible Navier-Stokes equation using a pseudo-spectral method. The particles are then added and tracked by using the equation of motion for the existing case, and a separate particle tracking method using the asymptotic expansion approach for particles with small Stokes number. The presence of the particles is assumed to not have any effect of the background turbulence as the volume fraction is of the order of $10^{-6}$. The smallest scales of the turbulence are defined by the Kolmogorov scales, namely, Kolmogorov length, velocity and time scale:

$$
\eta_{k}=\left(v^{3} / \epsilon\right)^{1 / 4} ; v_{k}=(v \epsilon)^{1 / 4} ; \tau_{k}=(\nu / \epsilon)^{1 / 2}
$$

where $\epsilon$ is the average viscous dissipation rate of the turbulence and $v$ is the air kinematic viscosity. The air viscosity is taken to be $v=0.17 \mathrm{~cm}^{2} / \mathrm{s}$, and air density, $\rho=0.001 \mathrm{gm} / \mathrm{cm}^{3}$. Another important parameter that characterizes the turbulence in cloud is the Taylor microscale Reynolds number $\left(R e_{\lambda}\right)$. It is defined as:

$$
R e_{\lambda}=u^{\prime} \lambda / \nu
$$

where $\lambda$ is the transverse Taylor microscale, defined as:

$$
\lambda=u^{\prime} /\left\langle\left(\partial u_{1} / \partial x_{1}\right)^{2}\right\rangle^{1 / 2}=\left(15 v u^{\prime 2} / \epsilon\right)^{1 / 2}
$$

and $u^{\prime}$ is the r.m.s. fluctuation velocity in a given direction. For the direct integration approach, the droplets are driven by the Stokes drag, body force, and inertia:

$$
\frac{d V(t)}{d t}=\frac{U(Y(t), t)-V}{\tau_{p}}+g
$$

where $V$ is the velocity of the particle, $U(Y(t), t)$ is the fluid velocity at the particle location. It is calculated in the code by a six-point Lagrangian interpolation technique, $g$ is the acceleration due to gravity, $\tau_{p}$ is the particle inertial response time (the time taken by the particle to react to a change in the flow) and, under Stokes drag it is given by:

$$
\tau_{p}=\frac{2}{9} \frac{\rho_{w}}{\rho}\left(\frac{a}{\eta}\right)^{2} \tau_{k}
$$


where $\rho_{w}=1 \mathrm{gm} / \mathrm{cm}^{3}$ is the density of the particles (water), and $a$ is the particle radius. The still fluid settling velocity of the particle is determined by the equation:

$$
v_{p}=\tau_{p}|g|
$$

The Stokes number is defined as the ratio of the particle inertial response time to the smallest time scale of the turbulence (Kolmogorov time-scale), and the non-dimensional settling velocity is defined as the ratio of the particle velocity to the Kolmogorov velocity scale:

$$
S t=\tau_{p} / \tau_{k} ; S v=v_{p} / v_{k}
$$

\subsection{Problem definition and specific considerations}

For strato-cumulus clouds, the dissipation rates are relatively lower than that of cumulus clouds (less than $\left.100 \mathrm{~cm}^{2} / \mathrm{s}^{3}\right)$, the droplet size range in strato-cumulus clouds is relatively smaller $(5-40 \mu \mathrm{m})$. Since the CFL condition determines the numerical stability of the spectral simulation of the turbulent flow, we use it to determine why the simulations are expensive, with a combination of low particle Stokes number and low flow dissipation rate. CFL number is:

$$
C F L=\frac{U_{\max } d t_{\text {flow }}}{d x}
$$

where $U_{\max }$ is the maximum flow velocity, $d t$ is the discretization time-step size, and $d x$ is the grid spacing. We set $U_{\max } \sim 5 u^{\prime}$, where $u^{\prime}$ is the r.m.s. fluctuation velocity, and in the spectral method for air turbulence simulation, generally, $d x=2 \eta$, where $\eta$ is the Kolmogorov length scale. In DNS, the CFL number is typically less than 0.25 . So, $d t$ becomes:

$$
\frac{d t_{\text {flow }}}{\tau_{k}} \sim \frac{1}{10} \frac{v_{k}}{u^{\prime}}
$$

Equation 9 can be alternatively written as:

$$
d t_{\text {flow }}=C F L \times \frac{2}{5} 15^{0.25} R e_{\lambda}^{-0.5} \sqrt{\frac{\nu}{\epsilon}}
$$

Combining equations 10 and 5, we obtain:

$$
\frac{d t_{\text {flow }}}{\tau_{p}}=\frac{0.8 \times C F L \times v^{1.5}}{\sqrt{R e_{\lambda}} \times \frac{2}{9} \times 1000 \times a^{2} \times \sqrt{\epsilon}}
$$

Equation 11, shows that with decreasing average flow dissipation rate $\epsilon$, and the particle radius, $a$, the particle inertial response time $\tau_{p}$ may become smaller than the time step $d t_{\text {flow }}$ needed for the flow simulation. Since the time step for integrating the particle velocity must be less than $\tau_{p}$, then in this case, the time step size is governed by $\tau_{p}$. So for low dissipation rates and small particle radius, as in the case of strato-cumulus clouds the $d t$ required is very small. Hence we need to run the simulations for more time steps to obtain reasonable average statistics with small uncertainty, making the simulations computationally very expensive.

\section{METHOD}

\subsection{Asymptotic expansion equation}

Let us start with the formal integral formulation of the equation of motion for a small heavy particle:

$$
\tau_{p} \frac{d V_{i}\left(t, \mathbf{Y}_{0}, \mathbf{V}_{0}\right)}{d t}=\left[u_{i}(\mathbf{Y}, t)-V_{i}+v_{p_{i}}^{S}\right]
$$




$$
\frac{d Y_{i}\left(t, \mathbf{Y}_{0}, \mathbf{V}_{0}\right)}{d t}=V_{i}\left(t, \mathbf{Y}_{0}, \mathbf{V}_{0}\right)
$$

where, $\tau_{p}$ is the particle inertial response time, $V_{i}(t)$ is the particle velocity, $u_{i}(\mathbf{x}, t)$ is the flow field, and $v_{p}{ }_{i}^{S}$ is the constant particle settling velocity in still fluid. $\mathbf{Y}_{0}$ and $\mathbf{V}_{0}$ are particle initial position and initial velocity at $t=0$. Using the small inertia limiting value, and a small particle response time we integrate the equation, and perform a double Taylor expansion on $u(x, t)$, with respect to $(x=Y(t), t)$. Finally, we get,

$$
V_{i}(t ; \mathbf{Y}(t)=\mathbf{x})=v_{p_{i}}^{S}+u_{i}(\mathbf{x}, t)-\tau_{p}\left[\frac{D u_{i}}{D t}+v_{p_{j}} \frac{\partial u_{i}}{\partial x_{j}}\right]+\mathcal{O}\left(\tau_{p}^{2}\right)
$$

where

$$
\frac{D u_{i}}{D t} \equiv \frac{\partial u_{i}}{\partial t}+u_{j} \frac{\partial u_{i}}{\partial x_{j}}
$$

Equation 14 is the well-known result first obtained by Maxey (1987, Eq. (5.7))[9], and Eq. (1) in Balachandar and Eaton (2010) [10]. This is the equation we use for the asymptotic expansion method in our simulations.

\subsection{Turbulent Flow}

The solver is an in-house code based on Direct Numerical Simulation (DNS). For the turbulent flow, we generate the fluid turbulence using a pseudo-spectral method in a periodic domain, by solving direct numerical simulation of the Navier-Stokes equation:

$$
\frac{\partial \vec{U}}{\partial t}=\vec{U} \times \omega-\nabla\left(\frac{P}{\rho}+\frac{1}{2} \vec{U}^{2}\right)+v \nabla^{2} \vec{U}+\vec{f}(\vec{x}, t)
$$

and the continuity equation:

$$
\nabla \cdot \vec{U}(\vec{x}, t)=0
$$

where $\vec{U}$ is the flow-field $\omega=\nabla \times \vec{U}$ is the vorticity, $P$ is the pressure. We re-scale the cloud problem in DNS in a periodic box of length of $L_{b}=2 \pi=6.283$ (in DNS units), and match the liquid water content (LWC) with the cloud. LWC is the mass of liquid per unit volume, this quantity determines the number of particles present in the system, a lower value of LWC means less number of particles, and higher value signifies larger number of particles. Typically in strato-cumulus clouds, the LWC is about $1 \mathrm{gm} / \mathrm{m}^{3}$. The second parameter we try to match is the dissipation rate. In these simulations, we use weak values of turbulent dissipation rate as our goal is to use the asymptotic expansion for the specific case of combination of small particle size (we use $10 \mu \mathrm{m}-20 \mu \mathrm{m}$ particle pair) and dissipation rate of $\epsilon=10 \mathrm{~cm}^{2} / \mathrm{sec}^{3}$.

\section{RESULTS AND DISCUSSION}

\subsection{Validation of the asymptotic expansion for 2-D cellular flow}

We use the asymptotic expansion technique for particle tracking, first for a simple 2-D cellular flow (as in Maxey, 87 [9]). We use the results for this simpler flow to validate our implementation. The cellular flow is described as:

$$
\begin{gathered}
u_{1}=U_{0} \sin \left(x_{1}\right) \cos \left(x_{2}\right) \\
u_{2}=-U_{0} \cos \left(x_{1}\right) \sin \left(x_{2}\right)
\end{gathered}
$$

where $u$ is the flow velocity, $x$ is the position, and $L$ is the length of a cell, subscripts 1 , and 2 denote the $\mathrm{x}$ and $y$ directions, respectively. $2 \pi$ is the domain length in each direction. It should be noted that the fluid velocity in the particle location for this 2-D cellular flow is being directly calculated by using the position coordinates as opposed to the approximation by using the six point Lagrangian interpolation method for the turbulent flow. 
In this case we are using 4-cells in the domain, 2 in each direction $\mathrm{x}$ and $\mathrm{y}$, which makes the length of each cell to be $\pi$. Four point particles are introduced and the trajectories of the particles are shown in Figure 1. Two of the particle trajectories are approximated using the existing approach, and the other two of are approximated using the asymptotic approach. As expected in this case, the particles avoid the cell region (white porttion in the figure) in both approximation techniques. The particle Stokes number in all the cases is 0.03 . The particle

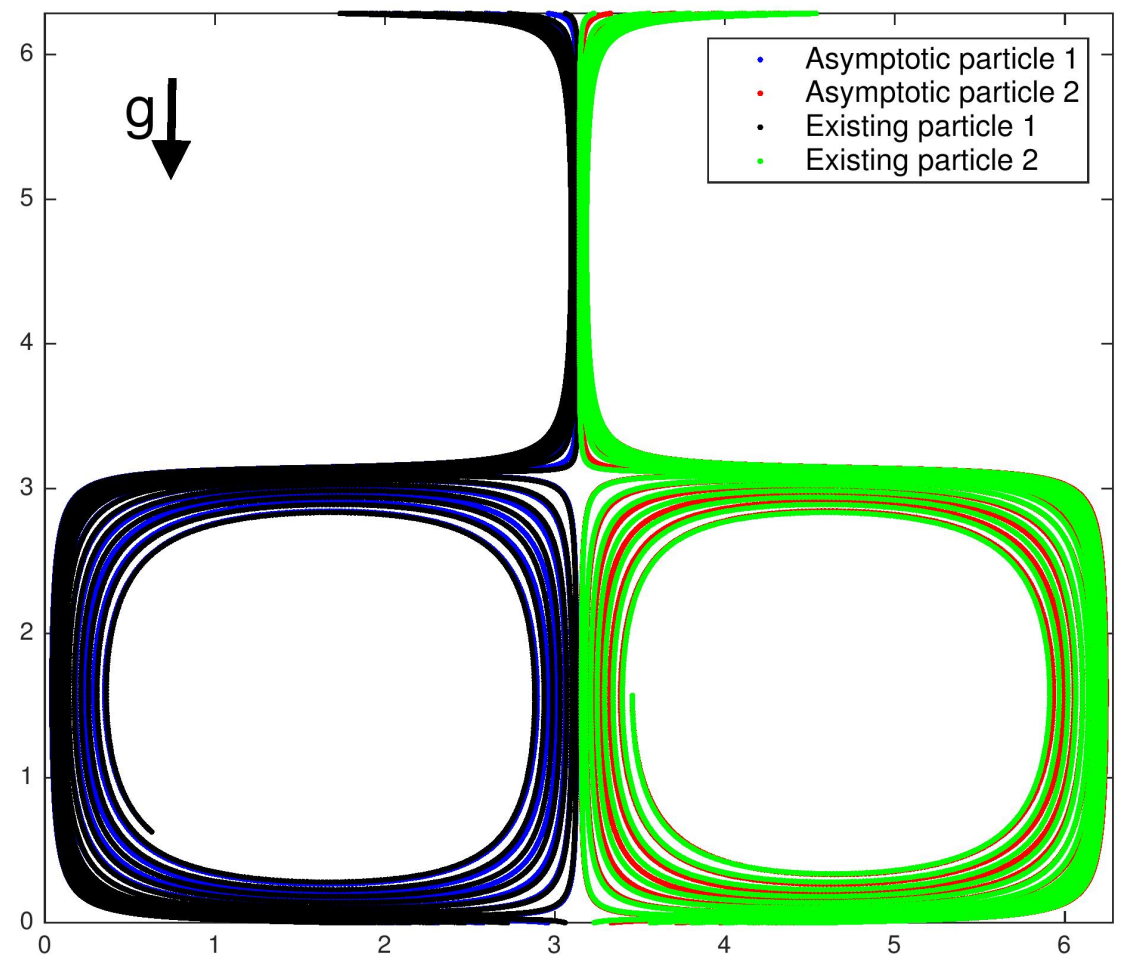

Fig. 1 Particle trajectory comparison for asymptotic and existing approach (direct integration approach) in 2D cellular flow

trajectories in both methods are similar, which validates the implementation of the asymptotic approach. We plot the relative error of $r=\sqrt{x^{2}+y^{2}}$ for the two methods as a function of time in figure 2. The error is normalized by the length of the domain $(2 \pi)$, and time is normalized by the particle response time $\tau_{p}$. The error can be concluded to be indeed very small. The reason for the semi-sinusoidal shape of the plot is that, when the particles are turning they are not in the exact same position according to both methods, so the changes in the $x$ and $y$ values are high at times, which makes the error value shoot up.

\subsection{Asymptotic expansion in turbulent flow}

We now use the asymptotic approach for a turbulent flow. Before we take a look at the results, we provide some information about the discretization time step size $d t$. The " $\mathrm{dt}$ " for the particle is given as $d t_{\text {particle }}=$ $10 \% \tau_{p}$, for the existing approach. So, the $d t_{\text {simulation }}$ is the minimum of $d t_{\text {flow }}$ and $d t_{\text {particle }}$. By using the asymptotic expansion, we can relax $d t_{\text {particle }}$ dependence to some extent, and use larger values, however the $d t_{\text {flow }}$ criteria remains same. In case of particles with low Stokes number, the $d t_{\text {particle }}$ is smaller than $d t_{\text {flow }}$. First we look at the comparison of the particle trajectories for a turbulent flow using the two approaches. Both simulations are for the same dissipation rate $\epsilon=10 \mathrm{~cm}^{2} / \mathrm{s}^{3}$, both particles are of same Stokes number, $S t=0.01$, and they have the same initial position. We set $d t_{\text {particle }}=10 \% \tau_{p}$, for the existing approach, 


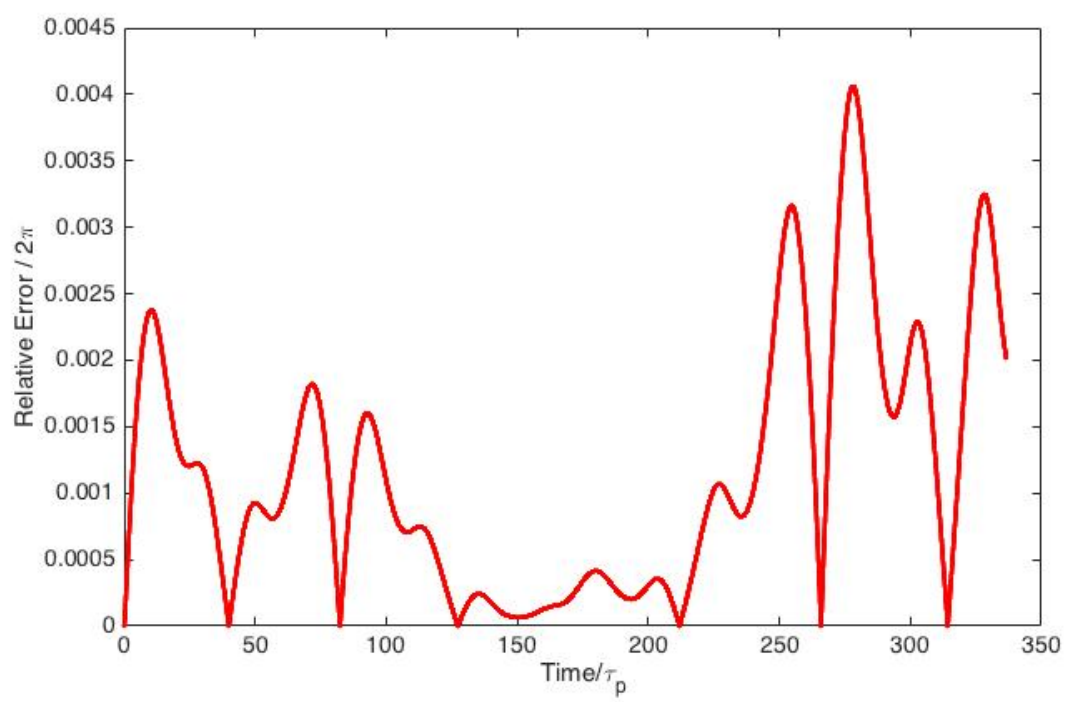

Fig. 2 Relative error of position between asymptotic and existing approach (direct integration approach) as a function of time

the $\tau_{p}$ chosen here is the smaller of the two particle sizes. For the asymptotic case, we plot trajectories by using larger $d t$ values $\left(d t=10 \%, 20 \%, 30 \% \tau_{p}\right)$. A comparison of a large time-step size $\left(d t=10 \% \tau_{k}\right)$ is also added. It should be noted that we plot the trajectories up to the point in time (roughly $25 \times \tau_{k}$ ) when both of the particles are in the domain. Figure 3 shows the $\mathrm{x}$ vs $\mathrm{y}$ plot, and figure 4 shows the $\mathrm{x}$ vs $\mathrm{z}$ plot.

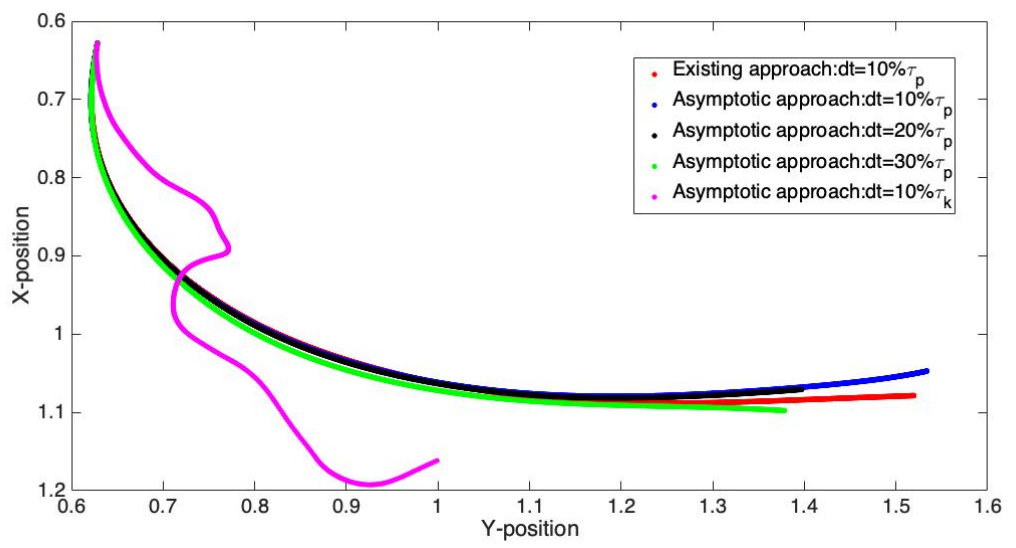

Fig. 3 Particle trajectory comparison for asymptotic and existing approach in turbulent flow (x vs y)

The trajectories for both methods look similar qualitatively, we do not expect an exact same trajectory in this case, as turbulent flow has a degree of randomness, and no two simulations would be exactly same. For the case of $d t=10 \% \tau_{k}$, we do expect a different trajectory, as the time-step size is very large in this compared to the others. We also plot the relative error in the r-direction in x-y plane (calculated by $r=\sqrt{x^{2}+y^{2}}$ ) in figure 5, and in $\mathrm{x}-\mathrm{z}$ plane (calculated by $r=\sqrt{x^{2}+z^{2}}$ ) in figure 6 . The errors shown in the figures are relative to the direct integration method with a $d t=10 \% \tau_{p}$. The asymptotic methods were simulated at three different $d t$ values, namely, $d t=10 \%, 20 \%, 30 \% \tau_{p}$, and $d t=10 \% \tau_{k}$. With increasing $d t$ values, larger error is expected. Next we compare the collision statistics obtained for the two methods. For the collision statistics, we run the simulations at a dissipation rate of $\epsilon=10 \mathrm{~cm}^{2} / \mathrm{s}^{3}$. The particle sizes are $10 \mu \mathrm{m}$ and $20 \mu \mathrm{m}$ in radius. The Stokes number are 0.01 and 0.04 respectively. We provide a comparison of the collision 


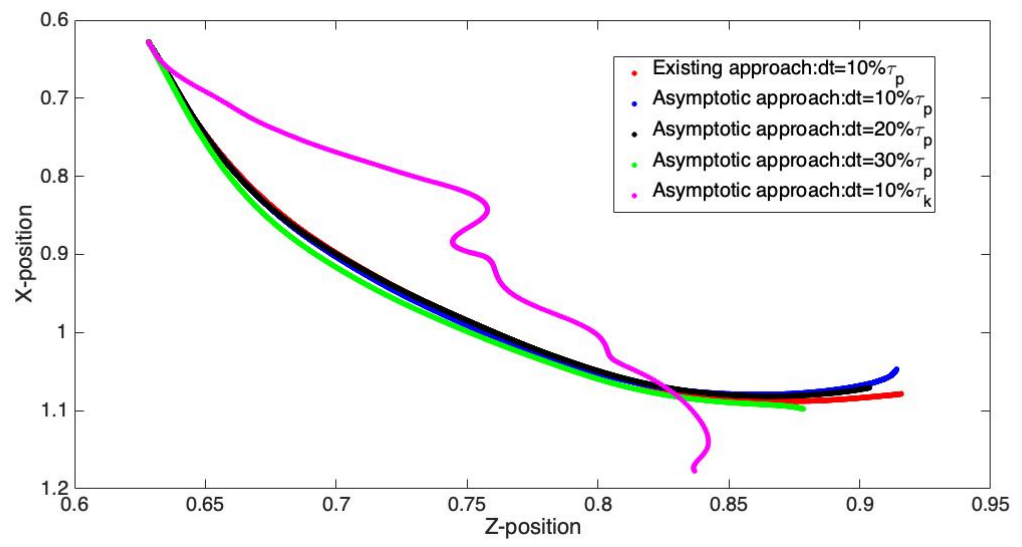

Fig. 4 Particle trajectory comparison for asymptotic and existing approach in turbulent flow (x vs z)

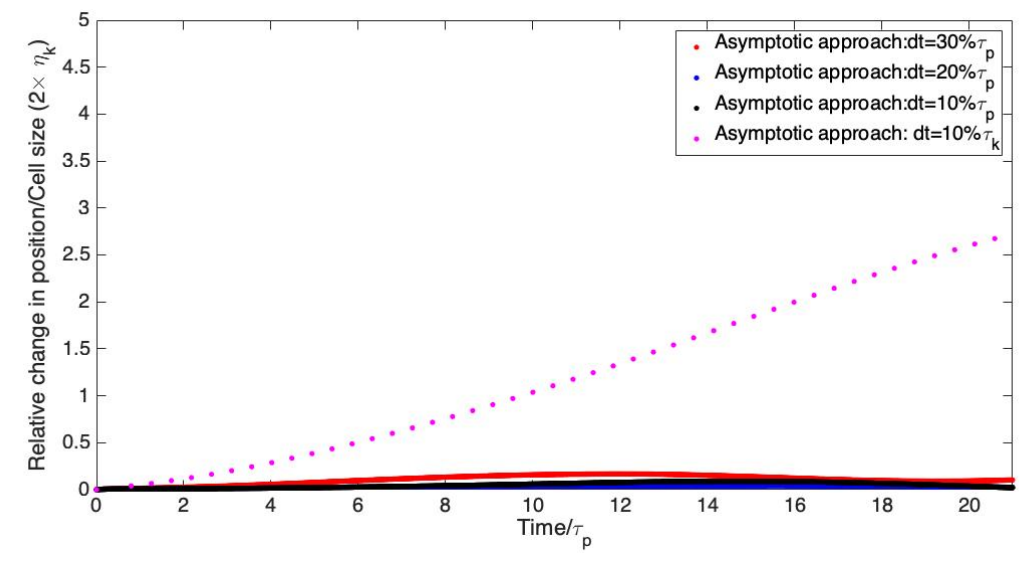

Fig. 5 Relative error of $r$ position (calculated by $r=\sqrt{x^{2}+y^{2}}$ ) as a function of time for asymptotic method compared to the direct integration method

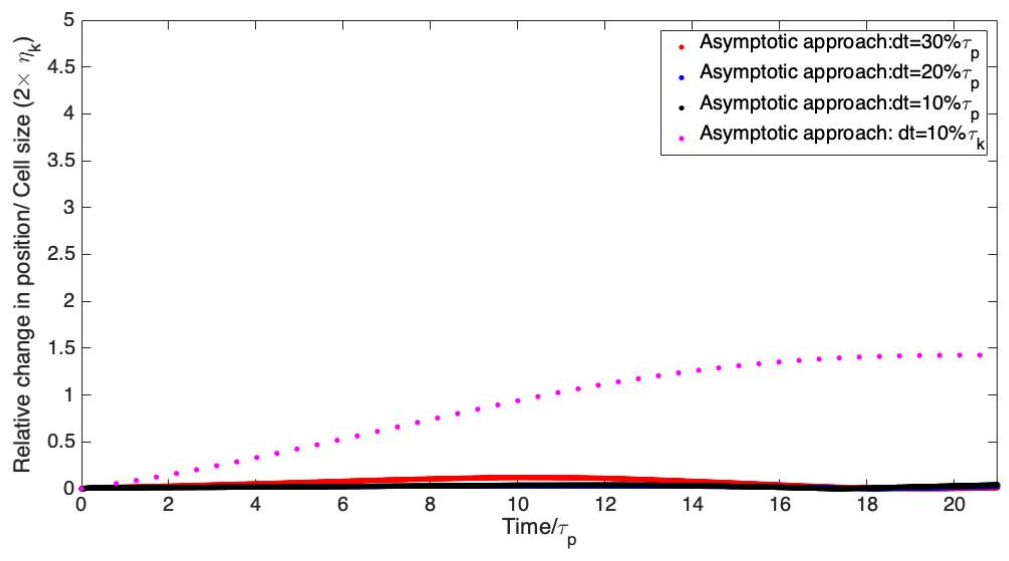

Fig. 6 Relative error of $r$ position (calculated by $r=\sqrt{x^{2}+z^{2}}$ ) as a function of time for asymptotic method compared to the direct integration method 
statistics for the existing approach of our simulations (with $d t=10 \% \tau_{p}$, based on $10 \mu m$ particle response time) to the asymptotic approach (for $d t=10 \%, 20 \%, 30 \%$ of $\tau_{p}$, and we provide a case of relatively large value of $d t$, where we set, $d t=10 \% \tau_{k}$, it should be noted that $\tau_{k} \sim 11.75 \times \tau_{p}$ ). We also provide the results for the same particle sizes, in the same flow dissipation rate as presented in Ayala et al 2008, [11], [12]). Table 1 provides the results for the cross-size mean relative velocity of particles at contact in the radial direction along with the standard deviation. The results for both approaches of our simulations is well within

Table 1 Comparison of $w_{r}$ for existing approach and asymptotic approach

\begin{tabular}{|c|c|c|}
\hline Case & $w_{r}$ & Standard Deviation \\
\hline Ayala et al, 2008 & 1.8927 & 0.0843 \\
\hline Existing $d t=10 \% \tau_{p}$ & 1.9228 & 0.0016 \\
\hline Asymptotic $d t=10 \% \tau_{p}$ & 1.9239 & 0.0016 \\
\hline Asymptotic $d t=20 \% \tau_{p}$ & 1.9234 & 0.0023 \\
\hline Asymptotic $d t=30 \% \tau_{p}$ & 1.9131 & 0.0029 \\
\hline Asymptotic $d t=10 \% \tau_{k}$ & 1.9022 & 0.0181 \\
\hline
\end{tabular}

the uncertainty limit of the published results in Ayala et al [11], [12]. In table 2 we provide a comparison for the cross-size radial distribution function (RDF), and its standard deviation for the same simulations. The

Table 2 Comparison of RDF, $(g(r))$ for existing approach and asymptotic approach

\begin{tabular}{|c|c|c|}
\hline Case & $g(r)$ & Standard Deviation \\
\hline Ayala et al, 2008 & 0.945 & 0.076 \\
\hline Existing $d t=10 \% \tau_{p}$ & 1.0034 & 0.0015 \\
\hline Asymptotic $d t=10 \% \tau_{p}$ & 0.9996 & 0.0015 \\
\hline Asymptotic $d t=20 \% \tau_{p}$ & 0.9980 & 0.0021 \\
\hline Asymptotic $d t=30 \% \tau_{p}$ & 1.0016 & 0.0026 \\
\hline Asymptotic $d t=10 \% \tau_{k}$ & 1.0092 & 0.0065 \\
\hline
\end{tabular}

results show very little difference with the published results in Ayala et al, 2008. There is little difference between the direct integration approach and the asymptotic approach. Finally, we present the results for the collision-kernel. Table 3 shows the comparison for the cross-size collision kernel and its standard deviation for the same simulations. The percentage difference of the mean collision kernel from our case is less than

Table 3 Comparison of collision kernel, $(\Gamma)$ for existing approach and asymptotic approach

\begin{tabular}{|c|c|c|}
\hline Case & $\Gamma$ & Standard Deviation \\
\hline Ayala et al, 2008 & $1.151 \mathrm{E}-04$ & $4.01 \mathrm{E}-06$ \\
\hline Existing $d t=10 \% \tau_{p}$ & $1.0911 \mathrm{E}-04$ & $2.61 \mathrm{E}-07$ \\
\hline Asymptotic $d t=10 \% \tau_{p}$ & $1.0875 \mathrm{E}-04$ & $2.61 \mathrm{E}-007$ \\
\hline Asymptotic $d t=20 \% \tau_{p}$ & $1.0855 \mathrm{E}-04$ & $3.69 \mathrm{E}-07$ \\
\hline Asymptotic $d t=30 \% \tau_{p}$ & $1.0835 \mathrm{E}-04$ & $4.51 \mathrm{E}-07$ \\
\hline Asymptotic $d t=10 \% \tau_{k}$ & $1.0856 \mathrm{E}-04$ & $2.83 \mathrm{E}-06$ \\
\hline
\end{tabular}

$5 \%$ to that of Ayala et al's results. It should be noted that the standard deviation of Ayala et al is higher, which when brought into consideration will bring down the percentage difference to an even lower value. Thus, we can conclude that our results are similar to that of Ayala et al. 


\subsection{Run time comparison}

Table 4 shows a comparison of the run time of the existing approach with asymptotic approach for the different $d t$ values, in a flow of same dissipation rate, $\epsilon=10 \mathrm{~cm}^{2} / \mathrm{s}^{3}$. The total physical time for simulation is same for all the cases, so the number of time-steps varies. The results show that the asymptotic expansion method of

Table 4 Comparison of run time for existing approach and asymptotic approach

\begin{tabular}{|c|c|}
\hline Case & Run Time (in minutes) \\
\hline Existing $d t=10 \% \tau_{p}$ & 972.67 \\
\hline Asymptotic $d t=10 \% \tau_{p}$ & 1278.43 \\
\hline Asymptotic $d t=20 \% \tau_{p}$ & 637.38 \\
\hline Asymptotic $d t=30 \% \tau_{p}$ & 429.01 \\
\hline Asymptotic $d t=10 \% \tau_{k}$ & 17.83 \\
\hline
\end{tabular}

particle tracking could be used as an alternative to the existing approach for particles with low Stokes numbers. The run time comparison shows that with relaxing $d t$, we can speed up the code considerably, although it takes more time for the asymptotic expansion for the same number of time-steps as in the existing method due to some extra interpolations of the fluid velocity derivatives at the particle position, the relaxation of the $d t$ enables us to reduce time eventually, which is the main advantage of the asymptotic expansion approach. Both existing method and asymptotic expansion method are approximations. Each method has a different way of approximating. For the specific case of small particle response time in weak turbulence, the asymptotic expansion generates results with similar accuracy to the existing method quickly as the discretization timestep size can be relaxed with the asymptotic expansion method, but in case of existing method it is stiff. Hence, with the existing method the simulations are computationally expensive, and that is the major reason for the application of the Asymptotic Expansion. The goal is achieve the collision statistics with similar accuracy at a faster time. However, it should be noted that with increasing particle Stokes Number, the accuracy of the asymptotic expansion technique would begin to reduce and the results would be much more different than the existing method. So, asymptotic expansion is only valid for small Stokes Number problem. The computational time taken in the existing approach is the major concern addressed in this article.

\section{CONCLUSIONS}

So far very little work has been done specific to cases where the dissipation rate is relatively low. As the low dissipation rate simulations are very expensive computationally, we implement the asymptotic expansion approach for particle tracking. For particles with small Stokes number the use of asymptotic expansion is valid. We use the asymptotic expansion equation for particle tracking as used by [9]. We validate our implementation by first using the approach for a simpler 2D cellular flow, and present the results for the particle trajectories for both methods of particle tracking. Next we compare particle trajectories for a turbulent flow using both methods. We compare the simulated collision statistics between the two methods, and with published results [11],[12]. The results show that the asymptotic expansion indeed produces reasonable results for the collision statistics. Finally, we compare the CPU times of the two methods, and show the speed up using the asymptotic expansion as we can relax the $d t$ size. So far we ran simulations for $d t=10 \% \tau_{k}$. It would also be interesting to determine the accuracy of the asymptotic expansion in case of larger particle sizes. The limiting value of the particle size for which the asymptotic expansion would work can be regarded as a future work, and it would be a step forward in the analysis of small particle tracking simulations in weak turbulence cases, as the bottleneck due to the expensive nature of the simulations would be reduced to a considerable extent. 
TFEC-2020-32308

\section{ACKNOWLEDGMENTS}

The authors acknowledge the NSF and NCAR grants, and the NCAR Yellowstone super-computer computational resources

\section{NOMENCLATURE}

a Particle Radius

$g \quad$ Acceleration due to gravity

$g(r) \quad$ Radial Distribution Function

$P \quad$ Pressure

$R e_{\lambda}$ Taylor Microscale Reynolds Number

$U \quad$ Fluid Velocity

$u \quad$ Flow field

$u^{\prime} \quad$ R.M.S. Fluctuation Velocity

$V \quad$ Particle Velocity

$v_{p} \quad$ Still fluid settling velocity of particle

$v_{k} \quad$ Kolmogorov Velocity Scale

$w_{r} \quad$ Relative Velocity

$x \quad$ Position Vector

$Y \quad$ Particle Location

$\begin{array}{ll}\eta_{k} & \text { Kolmogorov Length Scale } \\ \epsilon & \text { Turbulence Dissipation Rate } \\ \lambda & \text { Transverse Taylor Microscale } \\ \omega & \text { Vorticity } \\ \nu & \text { Viscosity } \\ \rho & \text { Density } \\ \tau_{k} & \text { Kolmogorov Time Scale } \\ \tau_{p} & \text { Particle Response Time }\end{array}$

\section{REFERENCES}

[1] Shivshankar Sundaram and Lance R. Collins. Collision statistics in an isotropic particle-laden turbulent suspension. part 1. direct numerical simulations. Journal of Fluid Mechanics, 335:75109, 1997.

[2] Lian-Ping Wang, Anthony S. Wexler, and Yong Zhou. On the collision rate of small particles in isotropic turbulence. i. zeroinertia case. Physics of Fluids, 10(1):266-276, 1998.

[3] Lian-Ping Wang, ANTHONY S. WEXLER, and Yong Zhou. Statistical mechanical descriptions of turbulent coagulation of inertial particles. Journal of Fluid Mechanics, 415:117-153, 072000.

[4] Fausto Carlos De Almeida. The collisional problem of cloud droplets moving in a turbulent environinentpart i: A method of solution. Journal of the Atmospheric Sciences, 33(8):1571-1578, 1976.

[5] M. Pinsky, A. Khain, and M. Shapiro. Collisions of small drops in a turbulent flow. part i: Collision efficiency. problem formulation and preliminary results. Journal of the Atmospheric Sciences, 56(15):2585-2600, 1999.

[6] H. R. Pruppacher and J. D. Klett. Microphysics of clouds and precipitation. Springer, 1997.

[7] A. S. Koziol and H. G. Leighton. The effect of turbulence on the collision rates of small cloud drops. Journal of the Atmospheric Sciences, 53(13):1910-1920, 1996.

[8] A. P. Khain and M. B. Pinsky. Turbulence effects on the collision kernel. ii: Increase of the swept volume of colliding drops. Quarterly Journal of the Royal Meteorological Society, 123(542):1543-1560, 1997.

[9] M. R. Maxey. The motion of small spherical particles in a cellular flow field. The Physics of Fluids, 30(7):1915-1928, 1987.

[10] S. Balachandar and John K. Eaton. Turbulent dispersed multiphase flow. Annual Review of Fluid Mechanics, 42(1):111-133, 2010.

[11] Orlando Ayala, Bogdan Rosa, Lian-Ping Wang, and Wojciech W Grabowski. Effects of turbulence on the geometric collision rate of sedimenting droplets. part 1. results from direct numerical simulation. New Journal of Physics, 10(7):075015, jul 2008.

[12] Orlando Ayala, Bogdan Rosa, and Lian-Ping Wang. Effects of turbulence on the geometric collision rate of sedimenting droplets. part 2. theory and parameterization. New Journal of Physics, 10(7):075016, jul 2008. 